Check for updates

Cite this: RSC Adv., 2017, 7, 31502

\title{
Self-assembly fabrication, microstructures and antibacterial performance of layer-structured montmorillonite nanocomposites with cationic silica nanoparticles
}

\begin{abstract}
Xiangshuo Wang, ${ }^{a}$ Ling Shi, ${ }^{\star a}$ Junying Zhang, ${ }^{a}$ Jue Cheng ${ }^{a}$ and Xiaodong Wang (D) ${ }^{b}$
This paper reports the synthesis and antibacterial performance of a novel type of layer-structured montmorillonite (MMT) nanocomposite with cationic silica nanoparticles through an intercalating selfassembly method. The quaternary amine-functionalized $\mathrm{SiO}_{2}$ nanoparticles were first prepared by grafting dimethyloctadecyl [3-(trimethoxysilyl) propyl] ammonium chloride (PQAC) onto the surfaces of silica nanoparticles, and their chemical composition and structure were characterized by Fouriertransform infrared spectroscopy, thermogravimetric analysis and X-ray photoelectron spectroscopy. Then, the reactant $P Q A C-\mathrm{SiO}_{2}$ nanoparticles were intercalated into the interlayers of $M M T$ through electrostatic self-assembly to achieve the layer-structured MMT/PQAC-SiO 2 nanocomposites. The ordered and layered structural characteristics of the resulting nanocomposites were characterized using $\mathrm{X}$-ray diffraction patterns and $\mathrm{N}_{2}$ adsorption-desorption measurements and then confirmed by scanning electron microscopy and transmission electron microscopy. The optimum weight ratio of MMT/PQAC$\mathrm{SiO}_{2}$ nanoparticles was also achieved for the self-assembly fabrication of the nanocomposites on the basis of results mentioned above. The MMT/PQAC-SiO 2 nanoparticles gained an excellent thermal stability due to the intercalation of PQAC- $\mathrm{SiO}_{2}$ nanocomposites into MMT and their onset degradation temperature was improved by $40{ }^{\circ} \mathrm{C}$ compared to that of $\mathrm{PQAC}-\mathrm{SiO}_{2}$ nanoparticles. Most of all, the nanocomposites presented a significant antibacterial effect against Escherichia coli and Staphylococcus aureus as the model microorganisms of Gram-negative and Gram-positive bacteria, respectively. This type of layered structural nanocomposite is expected to be applied for food packaging and containment of food materials, medical bandages for wound care, removal of environment pollutants like pesticides and phenol, etc.
\end{abstract}

Received 18th April 2017

Accepted 8th June 2017

DOI: $10.1039 / \mathrm{c} 7 \mathrm{ra0} 4353 \mathrm{~h}$

rsc.li/rsc-advances

\section{Introduction}

Nanocomposites based on layer-structured silicate matrices and organic/inorganic compounds are currently one of the most interesting topics for the research and development of advanced functional materials and have received a great deal of attention from both scientific societies and industrial communities. ${ }^{1}$ Generally, clay as a sort of layer-structured silicate can be broadly classified into two categories: natural and synthetic clays. Natural clays are basically composed of alternating sheets of " $\mathrm{SiO}_{2}$ " and " $\mathrm{AlO}_{6}$ " units with different mass ratios, which include kaolinite, halloysite, rectorite, chrysotile, smectite, vermiculite, pyragonite, mica, and chlorite. ${ }^{2,3}$ Among these natural clays, montmorillonite (MMT) clay is a type of smectite

${ }^{a}$ Key Laboratory of Beijing City on Preparation and Processing of Novel Polymer Materials, Beijing University of Chemical Technology, Beijing 100029, China. E-mail: shiling@mail.buct.edu.cn; Fax: +86 106443 8296; Tel: +86 1064438296

${ }^{b}$ State Key Laboratory of Organic-Inorganic Composites, Beijing University of Chemical Technology, Beijing 100029, China with a structure of $2: 1$ phyllosilicates and has been attracting extensive interest in fundamental research and commercial development due to its high cationic exchange capacity, swelling capacity, high surface areas and consequential strong adsorption capacities as well as its broad applications in the preparation of nanocomposites. ${ }^{4}$ The natural MMT with exchangeable cations can be transformed into hydrophobic organic MMT by replacing the metal ions with inorganic ones like silica and organic ones like quaternary ammonium salts., Such modified materials have a larger basal spacing between two layers as well as lower surface energy and can be compatible with organic liquids or polymers. Moreover, the change in the surface property influences the use of MMT clay in different areas. $^{7}$

There are numerous successful cases confirming that the hybrids of layered MMT clay and inorganic nanoparticles cannot only effectively enhance the intrinsic properties of inorganic nanoparticles but also improve the catalysis capability, catalysis capability, long-term thermal stability, high 
durability, and regenerability of the resulting nanocomposites. $^{8-10}$ Boutoumi et al. ${ }^{11}$ synthesized a kind of MMT/ $\mathrm{TiO}_{2} /$ polythiophene nanocomposites and found that it exhibited an enhanced degradation capability for the dye of rhodamine 6G. Mao et al. ${ }^{12}$ reported the synthesis of mesostructured aluminosilicate intercalates and their application as an acidic catalyst for catalytic cracking. Ruiz-Hitzky et al. ${ }^{\mathbf{1 3}}$ reported the preparation of a new class of hybrid materials based on the $\mathrm{Na}^{+}-$ $\mathrm{MMT} /$ chitosan bionanocomposites and found that such bionanocomposites have an anion-exchange capability suitable to build potentiometric sensors. Meanwhile, some of studies were focused on the further modification of clays by antibiotic constituents. Lin et al. ${ }^{\mathbf{1 4}}$ reported the immobilization of MMT with silver nanoparticles and found the resulting composites exhibited a mild inflammatory response and an outstanding antibacterial activity. Lavorgna et al. ${ }^{15}$ developed the active nanocomposite films consisting of layered MMT intercalated with chitosan and silver-nanoparticles through an ion exchange reaction, and they found that the films could release silver ions in a steady and prolonged manner. Although silver nanoparticles have a high antibacterial activity due to their large specific surface area, they can induce toxicity when used alone. This may result in a limitation to their application in broader areas. ${ }^{16}$ Zhou et al. ${ }^{17}$ reported a surface modification method for MMT by use of cationic gemini surfactants and found that these modified materials exhibit potential applications for removal of environment pollutants such as pesticides and for medical fields as antimicrobial materials. The MMT intercalated with quaternary ammonium compounds is well known as 'organoclays' and has been widely used as disinfectants and antiseptics in food processing environments. ${ }^{18}$

As a class of traditional antimicrobial agents, quaternary ammonium salts have been widely applied for preoperative disinfection of unbroken skin and non-critical surfaces, ${ }^{19}$ mucous membranes, ${ }^{20}$ and hand sanitizers. ${ }^{21}$ Although quaternary ammonium salts have a high antimicrobial efficiency and low antimicrobial resistance, ${ }^{22}$ they exhibit a non-controllable release behavior with a short-term antibacterial activity, and meanwhile, the unnecessary release of biocides can directly results in unpredictable long-term pollution. ${ }^{23}$ Moreover, quaternary ammonium salts are easily decomposed at a temperature higher than $150{ }^{\circ} \mathrm{C}$. The immobilization of quaternary ammonium salts onto the solid supporters like inorganic particles is considered as an effective and ecologically friendly method that can provide a long-term durability for this antimicrobial agent. ${ }^{24}$ Nowadays, the nanostructure silica materials have been accepted as a potential candidate supporter due to their characteristics of good biocompatibility, high thermal stability and easy surface modification. ${ }^{25-27}$ Hebalkar et $a .^{28}$ reported the synthesis of bi-functional silica particles through a simple wet chemical method by attaching titania and silver nanoparticles onto the surface of silica particles in a controlled way to form a core-shell structure, and their investigations indicated that the silica surface exhibited the efficient photo-catalytically self-cleaning and antibacterial activities. A new strategy for the preparation of mesoporous silica materials with different shapes was developed by Hao et al. ${ }^{29}$ and they found that the antibacterial activity of these mesoporous silica materials was dependent on their shapes. Qiu et $a l .{ }^{30}$ reported an electrostatic self-assembly method to prepare core-shell structural antimicrobial nanoparticles composed of silica and polyacrylamide and found that the resultant nanoparticles showed a stable and high antibacterial activity toward both $S$. aureus and $E$. coil.

In order to provide a long-term antibacterial capability and high thermal stability for quaternary ammonium salts, we attempted to employ MMT as a framework with a nanometric region and to use silica nanoparticles as a solid supporter to immobilize the polysiloxane quaternary ammonium chloride (PQAC) as an active ingredient in this work. It is highly expected that such the layer-structural immobilization can enhance the antimicrobial performance and thermal stability of PQAC due to the nanoscale effect in MMT interlayers. With this idea in mind, we first performed a chemical graft of PQAC as an antibacterial agent onto the surface of silica nanoparticles and then intercalated these PQAC-immobilized nanoparticles into MMT through an electrostatic self-assembly method. ${ }^{31,32}$ The microstructures of the resulting PQAC-silica/MMT nanocomposites were intensively studied, and their thermal and antibacterial properties were investigated. The aim of this study is to develop a novel type of MMT-based functional nanocomposites with an enhanced antibacterial activity and thermal stability.

\section{Experimental section}

\subsection{Materials}

Na-MMT clay with a cationic exchange capacity of 145 mequiv./ $100 \mathrm{~g}$ was commercially obtained from Nanocor Inc., USA. The colloidal silica nanoparticles (Ludox-SM) with a particle size of $7 \mathrm{~nm}$ were purchased from Sigma-Aldrich, USA, and they were suspended in water with a weight fraction of $30 \mathrm{wt} \%$. A solution of PQAC $\left[\left(\mathrm{CH}_{3} \mathrm{O}\right)_{3} \mathrm{Si}\left(\mathrm{CH}_{3}\right)_{3} \mathrm{~N}^{+}\left(\mathrm{CH}_{3}\right)_{2}\left(\mathrm{C}_{10} \mathrm{H}_{21}\right)\right]$ in methanol with a volume fraction of $60 \mathrm{vol} \%$ was commercially supplied by Acros Organics, Belgium. The other chemicals were purchased from Beijing Chemical Reagent Co., Ltd., China and used as received.

\subsection{Preparation methods}

A quaternization reaction was first performed for the colloidal silica nanoparticles. In a typical reaction process, $7 \mathrm{ml}$ of colloidal silica sol was diluted in $40 \mathrm{ml}$ of deionized water, and then the resulting suspension was added dropwise into $10 \mathrm{ml}$ of PQAC solution under magnetic agitation at room temperature. With the formation of a white precipitate, and the suspension was aged at room temperature with mild stirring for $24 \mathrm{~h}$. Sequentially, the supernatant liquid was discarded, and the precipitate was washed thoroughly with deionized water and methanol. The resultant PQAC-modified silica nanoparticles (marked as $\mathrm{PQAC}-\mathrm{SiO}_{2}$ ) were redispersed in deionized water with a weight fraction of $1.2 \mathrm{wt} \%$ for further use. ${ }^{33}$

A series of layer-structured nanocomposites with different mass ratios of $\mathrm{MMT} / \mathrm{PQAC}-\mathrm{SiO}_{2}$ were prepared through an intercalating self-assembly method. In a typical process, $1.6 \mathrm{~g}$ of 
MMT powders were dispersed in $80 \mathrm{ml}$ of deionized water, and then the resulting slurry was heated to reflux at $80^{\circ} \mathrm{C}$ for $6 \mathrm{~h}$ to make MMT powders fully swollen. The obtained suspension was centrifuged for 30 minutes, and then the suspension in the upper layer was collected with for the further intercalating selfassembly with PQAC- $\mathrm{SiO}_{2}$ nanoparticles. The suspension containing $\mathrm{PQAC}-\mathrm{SiO}_{2}$ nanoparticles was added to the swollen MMT suspension and then was stirred at $70{ }^{\circ} \mathrm{C}$ for $8 \mathrm{~h}$. Ultimately, the resultant $\mathrm{MMT} / \mathrm{PQAC}-\mathrm{SiO}_{2}$ nanocomposites were washed several times with deionized water and then dried at $70{ }^{\circ} \mathrm{C}$ for $24 \mathrm{~h}$.

\subsection{Characterization and measuring method}

Fourier-transform infrared (FTIR) spectroscopy was conducted on a Bruker Alpha-T FTIR spectrometer at a scanning number of 32 using a KBr sampling sheet. The thermal stability of nanocomposites was characterized by thermogravimetric analysis (TGA) on a TA Instruments SDT2960 thermogravimetric analyzer at a heating rate of $5{ }^{\circ} \mathrm{C} \min ^{-1}$ under a nitrogen atmosphere. The zeta potentials of the MMT and $\mathrm{PQAC}-\mathrm{SiO}_{2}$ suspensions were measured by a Malvern Zetasizer Nano-ZS ZEN 3600 zeta potential analyzer. X-ray photoelectron spectroscopy (XPS) analysis was performed with a VG Scientific ESCALAB 250 X-ray photoelectron spectrometer with a focused monochromatized $\mathrm{Al} \mathrm{K} \alpha$ radiation. The obtained XPS spectra were fitted for the Casa XPS software, in which a Shirley background was assumed. X-ray diffraction (XRD) powder patterns were obtained from a Rigaku D/Max $2500 \mathrm{VBZ}+/ \mathrm{PC}$ X-ray diffractometer using $\mathrm{Cu}-\mathrm{K} \alpha$ radiation and operated at $40 \mathrm{kV}$ and $50 \mathrm{~mA}$ with a scan rate of $1^{\circ} \mathrm{min}^{-1}$. The morphologies of nanocomposites were observed by scanning electron microscopy (SEM) using a JEOL JSM-7800 scanning electron microscope and their microstructures were characterized by transmission electron microscopy (TEM) on a FEI Tecnai G2 20 transmission electron microscope operating at an acceleration voltage of $200 \mathrm{kV}$. The $\mathrm{N}_{2}$ adsorption-desorption isotherms were measured at $77 \mathrm{~K}$ using a Quantachrome Autosorb-1 absorption analyzer with prior degassing under vacuum at $120{ }^{\circ} \mathrm{C}$ for $12 \mathrm{~h}$. The data of specific surface area was calculated from the adsorption branch of the isotherm by using a BarrettJoyner-Halenda (BJH) method.

The antimicrobial performance of $\mathrm{MMT} / \mathrm{PQAC}-\mathrm{SiO}_{2}$ nanocomposites was measured according to a standard plate count method as described by the ref. 34-36 Escherichia coli (E. coli) as a Gram-positive bacterium and Staphylococcus aureus (S. aureus) as a Gram-negative bacterium were commercially obtained from National Center for Medical Culture Collections and selected to antibacterial tests, and both of them contained 10 (ref. 6 and 7) colony forming units (CFU) per each milliliter. For a typical measuring process, $50 \mathrm{mg} \mathrm{ml}^{-1}$ of suspension containing MMT/ $\mathrm{PQAC}^{-\mathrm{SiO}_{2}}$ nanocomposites was mixed with $25 \mathrm{ml}$ of normal salon to evaluate the antibacterial activity. Prior to the formal measurement, these bacteria were cultivated in the LuriaeBertani medium for $24 \mathrm{~h}$. The cultivated bacteria were diluted and then added into the suspension containing MMT/PQAC$\mathrm{SiO}_{2}$ nanocomposites to achieve a viable cell concentration of about $10^{3} \mathrm{CFU} \mathrm{\textrm {ml } ^ { - 1 }}$. The bacteria solutions without nanocomposites were also prepared as controls. Aliquots $(100 \mu \mathrm{l})$ were withdrawn every $1 \mathrm{~h}$ to homogenously cast on a series of Petri dishes coated with the Luriae-Bertani agar medium. The plates were moved to the culturing chamber and incubated for $48 \mathrm{~h}$ at $37^{\circ} \mathrm{C}$. The bacterial colonies presented on the plate were imaged and counted. Each of nanocomposites was measured for each incubation time and each testing was performed in triplicate. Then, the average number of surviving bacterial colonies was obtained from the above test. The survival rates $(R)$ for the adherent $E$. coli and $S$. aureus in the medium were calculated by the following formula:

$$
R=\frac{C_{t}}{C_{0}} \times 100 \%
$$

where $C_{t}$ and $C_{0}$ present the average numbers of surviving bacterial colonies at the incubation time of 0 and $t$, respectively.

\section{Results and discussion}

\subsection{Structural characterizations of PQAC-SiO nanoparticles}

PQAC- $\mathrm{SiO}_{2}$ nanoparticles were synthesized through a polycondensation reaction between the silanol groups of silica nanoparticles and PQAC, and their chemical composition and structure were characterized by FTIR spectroscopy, and the resulting infrared spectra were illustrated in Fig. 1, where the spectra of pristine silica nanoparticles and PQAC were also presented as references. Pristine silica nanoparticles are found to exhibit two absorption peaks at 1109 and $475 \mathrm{~cm}^{-1}$ in their infrared spectrum, which are attributed to the $\mathrm{Si}-\mathrm{O}$ asymmetric and symmetric vibrations, respectively. ${ }^{30}$ Furthermore, an absorption band corresponding to the $\mathrm{Si}-\mathrm{O}$ bending vibration is also found at $799 \mathrm{~cm}^{-1}$. Compared to pristine silica nanoparticles, it can be observed that the infrared spectra of PQAC$\mathrm{SiO}_{2}$ nanoparticles show two new absorption bands at 1038 and $1135 \mathrm{~cm}^{-1}$ for the network structure formed by the $\mathrm{Si}-\mathrm{O}-\mathrm{Si}$

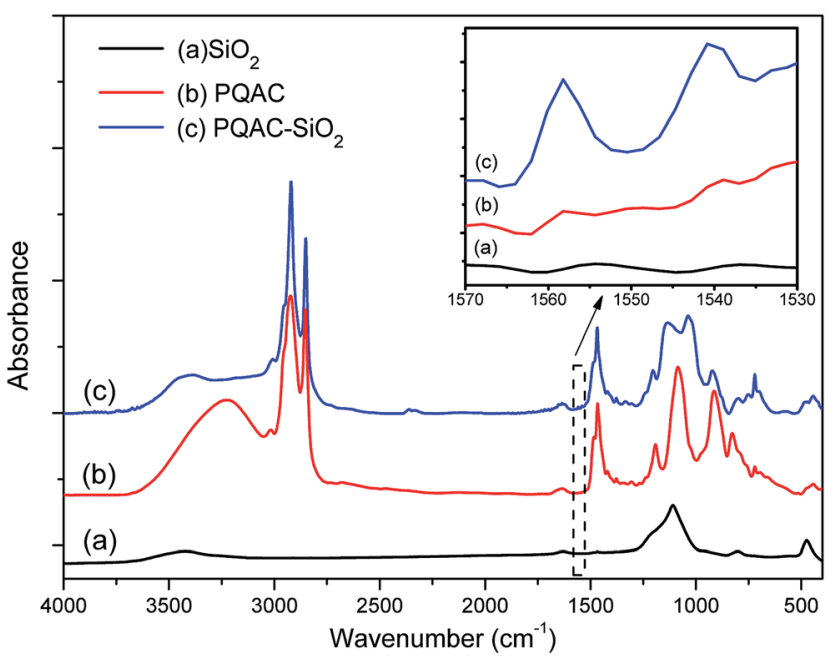

Fig. 1 FTIR spectra of pristine silica nanoparticles, PQAC and PQAC$\mathrm{SiO}_{2}$ nanoparticles. 
linkage, implicating that the hydrolyzed PQAC has condensed with the silanol groups on the surfaces of silica nanoparticles. Moreover, there are two characteristic absorption bands observed at 1558 and $1539 \mathrm{~cm}^{-1}$ due to the $\mathrm{C}-\mathrm{N}$ deformation vibration, ${ }^{37}$ and meanwhile, the other characteristic peaks also appears at $2920,2850,1470$, and $720 \mathrm{~cm}^{-1}$ in the infrared spectrum of PQAC-SiO $\mathrm{S}_{2}$ nanoparticles as observed in Fig. 1. These absorption bands are attributed to the stretching and scissoring deformation vibrations of methyl and methylene groups in PQAC, indicating that PQAC has been covalently grafted onto the silica nanoparticles. In addition, the appearance of a board band from $3200 \mathrm{~cm}^{-1}$ to $3600 \mathrm{~cm}^{-1}$ suggests that both PQAC and PQAC-SiO 2 nanoparticles contain an abundant of hydroxyl groups.

The surface elemental compositions of PQAC- $\mathrm{SiO}_{2}$ nanoparticles as well as the pristine silica ones were analyzed by XPS spectroscopy, and the obtained spectra were given in Fig. 2. As observe from Fig. 2, both pristine silica and $\mathrm{PQAC}-\mathrm{SiO}_{2}$ nanoparticles show the $\mathrm{O} 1 \mathrm{~s}, \mathrm{C} 1 \mathrm{~s}$ and $\mathrm{Si} 2 \mathrm{p}$ signals at binding energy of 532.4, 284.8 and $102.9 \mathrm{eV}$ in their survey XPS spectra of these two samples, respectively. It is noteworthy that the content of $\mathrm{C}$ atom for $\mathrm{PQAC}-\mathrm{SiO}_{2}$ nanoparticles increases significantly to $34.7 \%$ from $6.56 \%$ of pristine silica nanoparticles according to the signal intensity, whereas their content of $\mathrm{O}$ atom declines from $57.96 \%$ to $41.82 \%$. Moreover, the $\mathrm{N} 1 \mathrm{~s}$ signal appears at $402.45 \mathrm{eV}$ in the XPS spectrum of PQAC-SiO ${ }_{2}$ nanoparticles, which is due to the $\mathrm{NH}_{4}{ }^{+}$group derived from PQAC. ${ }^{38}$ Such a change in surface elemental composition confirms the existence of PQAC moieties on the surface of silica nanoparticles.

The thermal stabilities of $\mathrm{PQAC}-\mathrm{SiO}_{2}$ nanoparticles and PQAC were characterized by TGA. Fig. 3 shows the TGA and derivative TG thermograms of these two samples. PQAC is found to exhibit a typical two-step thermal degradation in the temperature ranges of $150-300{ }^{\circ} \mathrm{C}$ and $370-510{ }^{\circ} \mathrm{C}$ due to the decomposition of quaternary ammonium ions and the pyrolysis of alkane chains, ${ }^{39}$ respectively, and it also shows a char yield of $18.2 \mathrm{wt} \%$ at the end of decomposition. It seems that $\mathrm{PQAC}-\mathrm{SiO}_{2}$

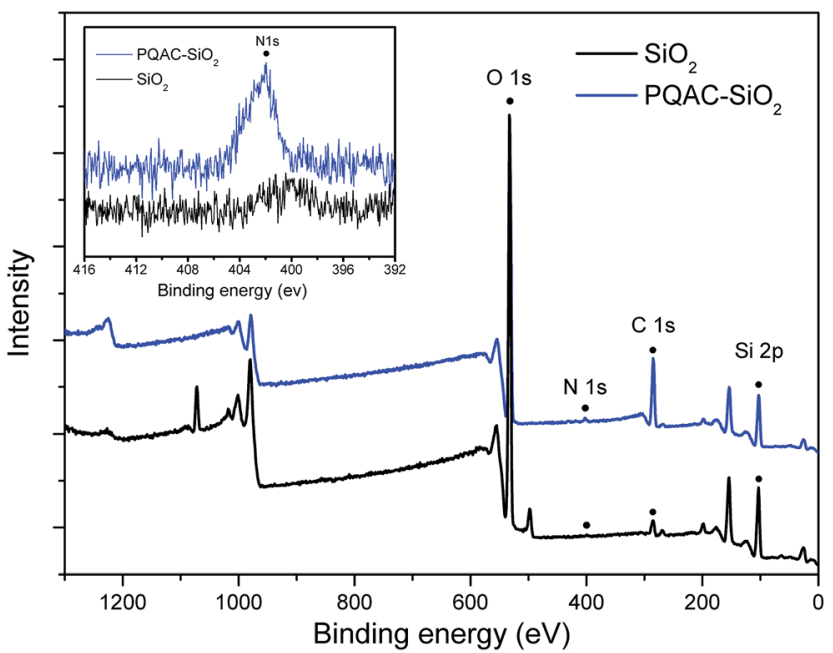

Fig. 2 XPS spectra of pristine silica nanoparticles and PQAC $-\mathrm{SiO}_{2}$ nanoparticles.

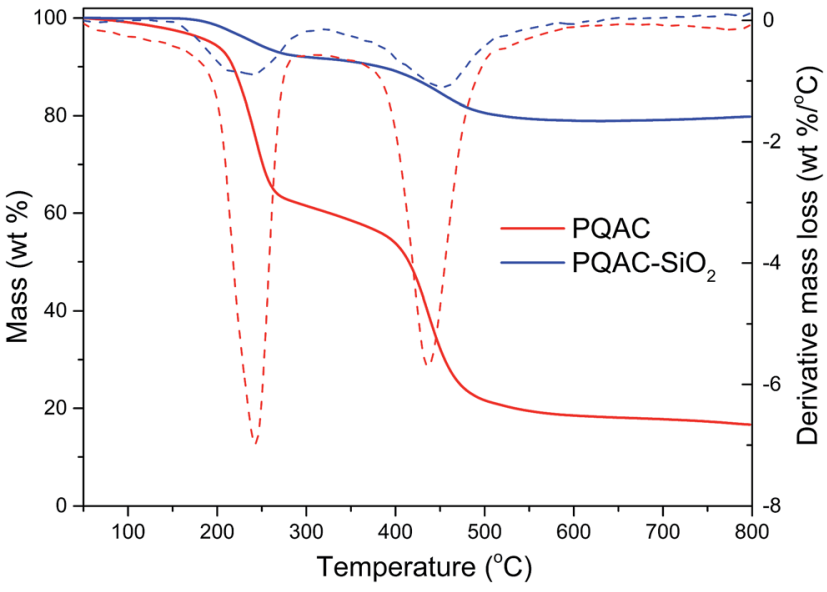

Fig. 3 TGA and DTG thermograms of silica nanoparticles, PQAC and $P Q A C-\mathrm{SiO}_{2}$ nanoparticles.

nanoparticles present a similar thermal degradation behavior with PQAC and, however, show a mass loss of $21.4 \mathrm{wt} \%$ at the end of thermal degradation. Such a mass loss can be attributed to the grafting ratio of PQAC on the silica nanoparticles.

\subsection{Preparation and structural characterizations of MMT/ PQAC-SiO 2 composites}

The MMT/PQAC-SiO ${ }_{2}$ composites were prepared through an intercalating electrostatic self-assembly method. This selfassembly mechanism is schematically illustrated in Fig. 4. It is expected that $\mathrm{PQAC}-\mathrm{SiO}_{2}$ nanoparticles are endowed with a positive charge on their surfaces through the functionalization with PAQC. On the other hand, MMT as a layer-structured material carries a permanent negative charge on the laminates, which has been balanced by the cationic conunterions within the interlayer space. In this case, positively charged $\mathrm{PQAC}-\mathrm{SiO}_{2}$ nanoparticles are introduced into the negatively charged MMT laminates, a rapid heterocoagulation takes place definitely due to multiple charge complex formation between two materials resulting from electrostatic interaction. ${ }^{40}$ Fig. 5 shows the zeta potentials of pristine silica nanoparticles, $\mathrm{PQAC}-\mathrm{SiO}_{2}$ nanoparticles and MMT with variation of $\mathrm{pH}$ vales of reactant

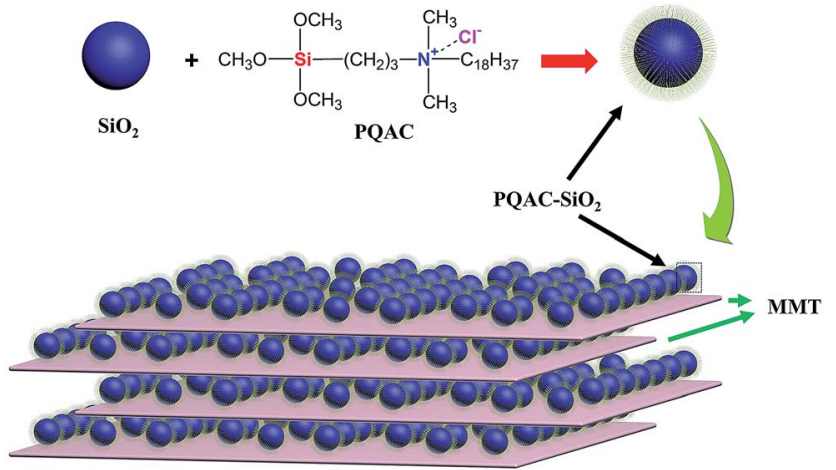

Fig. 4 Schematic

formation

process

of $\mathrm{MMT} / \mathrm{PQAC}-\mathrm{SiO}_{2}$ nanocomposites. 


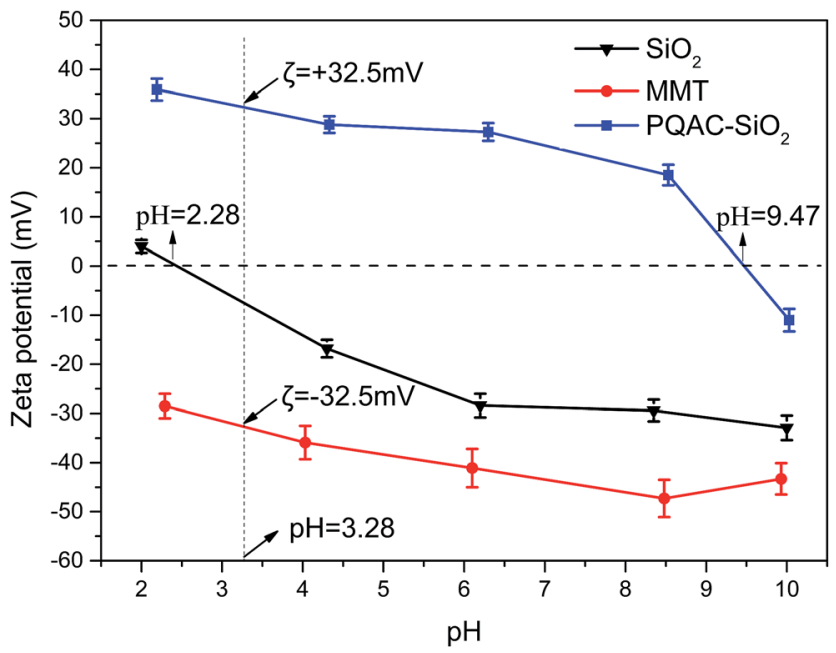

Fig. 5 Plots of zeta potential as a function of $\mathrm{pH}$ value for silica nanoparticles, MMT and PQAC- $\mathrm{SiO}_{2}$ nanoparticles in water.

suspension. It is observed in Fig. 5 that pristine silica nanoparticles shows a gradually decreasing negative zeta potential with an increase of $\mathrm{pH}$ value and also presents an isoelectric point at $\mathrm{pH}$ 2.28. However, the zeta potential of $\mathrm{PQAC}-\mathrm{SiO}_{2}$ nanoparticles exhibit a significant variation trend ranging from +35.9 to $-10.8 \mathrm{mV}$ with increasing the $\mathrm{pH}$ value of reactant suspension, and their isoelectric point was found at $\mathrm{pH}$ 9.47. On the other hand, MMT presents a negative zeta potential variation in the range of -28.5 to $-47.3 \mathrm{mV}$. In this case, it is easily determined that PQAC- $\mathrm{SiO}_{2}$ nanoparticles and MMT have the zeta potentials of +32.5 and $-32.5 \mathrm{mV}$, respectively, when the acidity of both of reactant suspensions are set to $\mathrm{pH}$ 3.2. Therefore, a maximum electrostatic interaction can be achieved at $\mathrm{pH} 3.2$ between these two materials when they are mixed. As a result, $\mathrm{PQAC}-\mathrm{SiO}_{2}$ nanoparticles are rapidly attracted into the MMT interlayers to form the highly ordered layer-structured nanocomposites.

Fig. 6 shows the XRD patterns of layer-structured MMT/ PQAC-SiO ${ }_{2}$ nanocomposites at different weight ratios. All of the nanocomposites are observed to exhibit a diffraction peak at $2 \theta$ $=7.3^{\circ}$ in their XRD patterns, which is assigned to the (001) reflection of MMT with a $d$-spacing of $1.2 \mathrm{~nm}$ determined by Bragg's law. It is well known that the pristine MMT layers can stack with one another by the attraction force between their cationic edges and anionic surfaces during the drying process due to their ionic nature, thus creating a $d$-spacing of 1.2$1.5 \mathrm{~nm}$. This phenomenon could be typically observed from various MMT-based materials and is associated with the water content of MMT. ${ }^{41}$ Nevertheless, it is noteworthy in Fig. 6a that this diffraction peak exhibits a gradual decline in its intensity with increasing the weight fraction of $\mathrm{PQAC}-\mathrm{SiO}_{2}$ nanoparticles, suggesting the gradual elimination of ordered structure in the (001) plane of MMT. Meanwhile, a new diffraction peak appears at $2 \theta=0.84^{\circ}$ in their XRD patterns, which corresponds to a $d$ spacing of $10.6 \mathrm{~nm}$ in the $\mathrm{MMT} / \mathrm{PQAC}-\mathrm{SiO}_{2}$ nanocomposites. Such a new diffraction peak is attributed to the formation of pillared structure supported by intercalating $\mathrm{PQAC}^{-\mathrm{SiO}_{2}}$
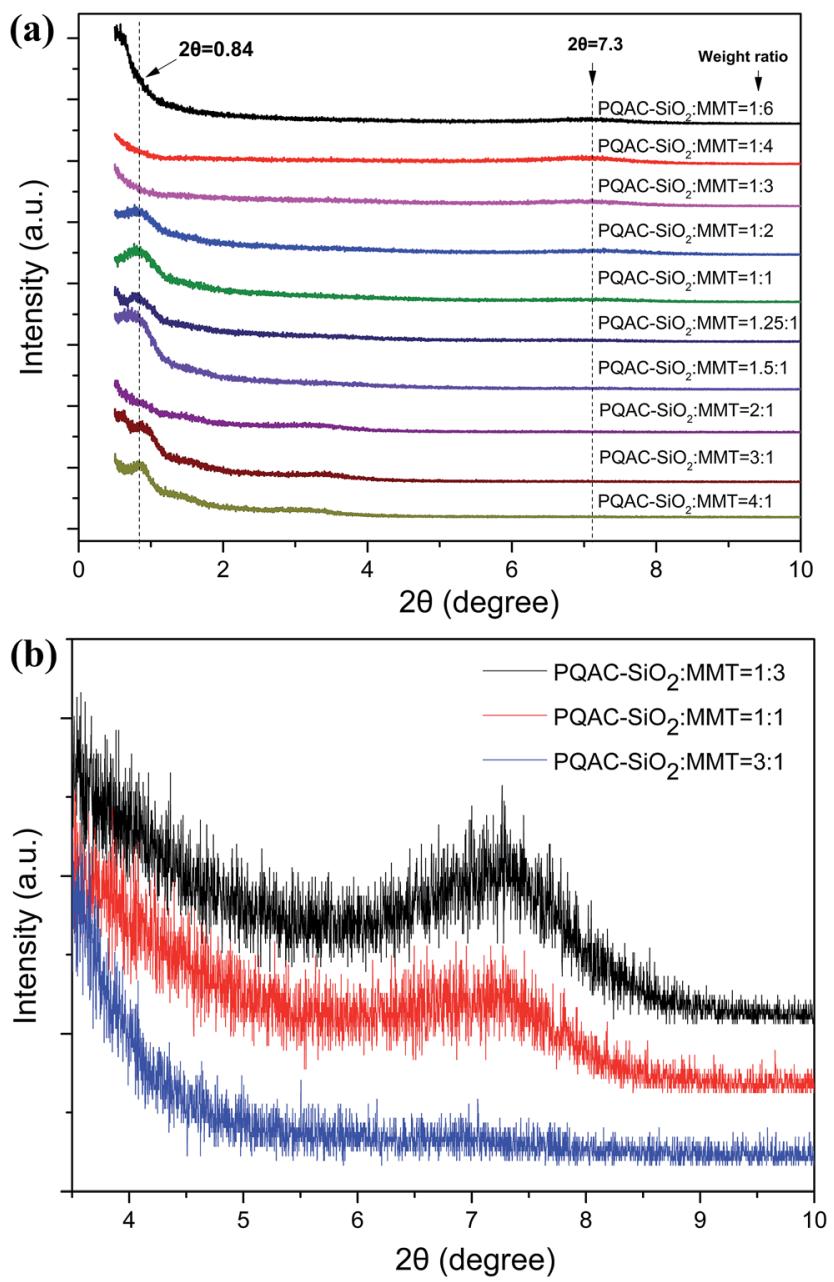

Fig. 6 X-ray diffraction patterns of $M M T / P Q A C-\mathrm{SiO}_{2}$ nanocomposites with different weights ratios of $M M T / P Q A C-\mathrm{SiO}_{2}$ nanocomposites.

nanoparticles into the interlayers of MMT, thus resulting in an enlarged interlayer space. Moreover, two diffraction peaks can be simultaneously observed from the $\mathrm{MMT} / \mathrm{PQAC}-\mathrm{SiO}_{2}$ nanocomposite at the weight ratio of $1 / 2$ as shown in Fig. 6 , indicating the coexistence of the original layered structure of MMT and the intercalation one formed by $\mathrm{PQAC}-\mathrm{SiO}_{2}$ nanoparticles. However, with continually increasing the weight fraction of PQAC-SiO ${ }_{2}$ nanoparticles, more and more $\mathrm{PQAC}-\mathrm{SiO}_{2}$ nanoparticles were intercalated into the interlayers of MMT until each of interlayers was full with the PQAC-SiO ${ }_{2}$ nanoparticles. In this case, the diffraction peak at $2 \theta=7.3^{\circ}$ is found to disappear at a high weight fraction of $\mathrm{PQAC}-\mathrm{SiO}_{2}$ nanoparticles, whereas the peak at $2 \theta=0.84^{\circ}$ is noticeably enhanced as seen in Fig. 6b. The interlayer space of $\mathrm{MMT} / \mathrm{PQAC}-\mathrm{SiO}_{2}$ nanocomposites could be determined as $9.4 \mathrm{~nm}$ by the new diffraction data according to Bragg's law, and meanwhile, the size of PQAC- $-\mathrm{SiO}_{2}$ nanoparticles could also be estimated as $9.4 \mathrm{~nm}$.

The morphologies and microstructures of MMT/PQAC-SiO ${ }_{2}$ nanocomposites were observed by SEM and then confirmed by TEM. Fig. 7 shows the SEM micrographs of layer-structured nanocomposites at different $\mathrm{MMT} / \mathrm{PQAC}-\mathrm{SiO}_{2}$ weight ratios. It 
is clearly observed in Fig. 7a-d that PQAC-SiO $\mathrm{S}_{2}$ nanoparticles are homogenously dispersed on the surfaces of MMT lamellae due to the electrostatic self-assembly, and most of them are monodispersed. Furthermore, the distribution of nanoparticles seems to become denser with an increase of weight fraction of PQAC- $\mathrm{SiO}_{2}$ nanoparticles. However, there are some agglomerated nanoparticles observed from Fig. 7a-d due to the overloading of $\mathrm{PQAC}-\mathrm{SiO}_{2}$ nanoparticles in MMT. On the other hand, the TEM micrographs in Fig. 8 confirm the uniform distribution of PQAC- $\mathrm{SiO}_{2}$ nanoparticles within the interlays of MMT. $^{42}$ These nanoparticles are observed to exhibit a regular array between the MMT lamellae, and the boundary between the nanoparticles is easily distinguished without overlapping because of their cationic nature. These results convincingly confirm that the formation of layer-structured nanocomposites based on multilayered MMT and PQAC-SiO ${ }_{2}$ nanoparticles.

The layer-structural characteristics of $\mathrm{MMT} / \mathrm{PQAC}-\mathrm{SiO}_{2}$ nanocomposites were further analyzed by $\mathrm{N}_{2}$ adsorptiondesorption experiments. Fig. 9 shows the representative $\mathrm{N}_{2}$ adsorption-desorption isotherms of $\mathrm{MMT} / \mathrm{PQAC}-\mathrm{SiO}_{2}$ nanocomposites as well as their specific surface areas calculated by the $\mathrm{BJH}$ method. As displayed in Fig. 9a, the PQAC-SiO $\mathrm{Pano}_{2}$ nano particles are found to present a typical Type IV isotherm with an H2 hysteresis loop according to the Brunauer-Deming-
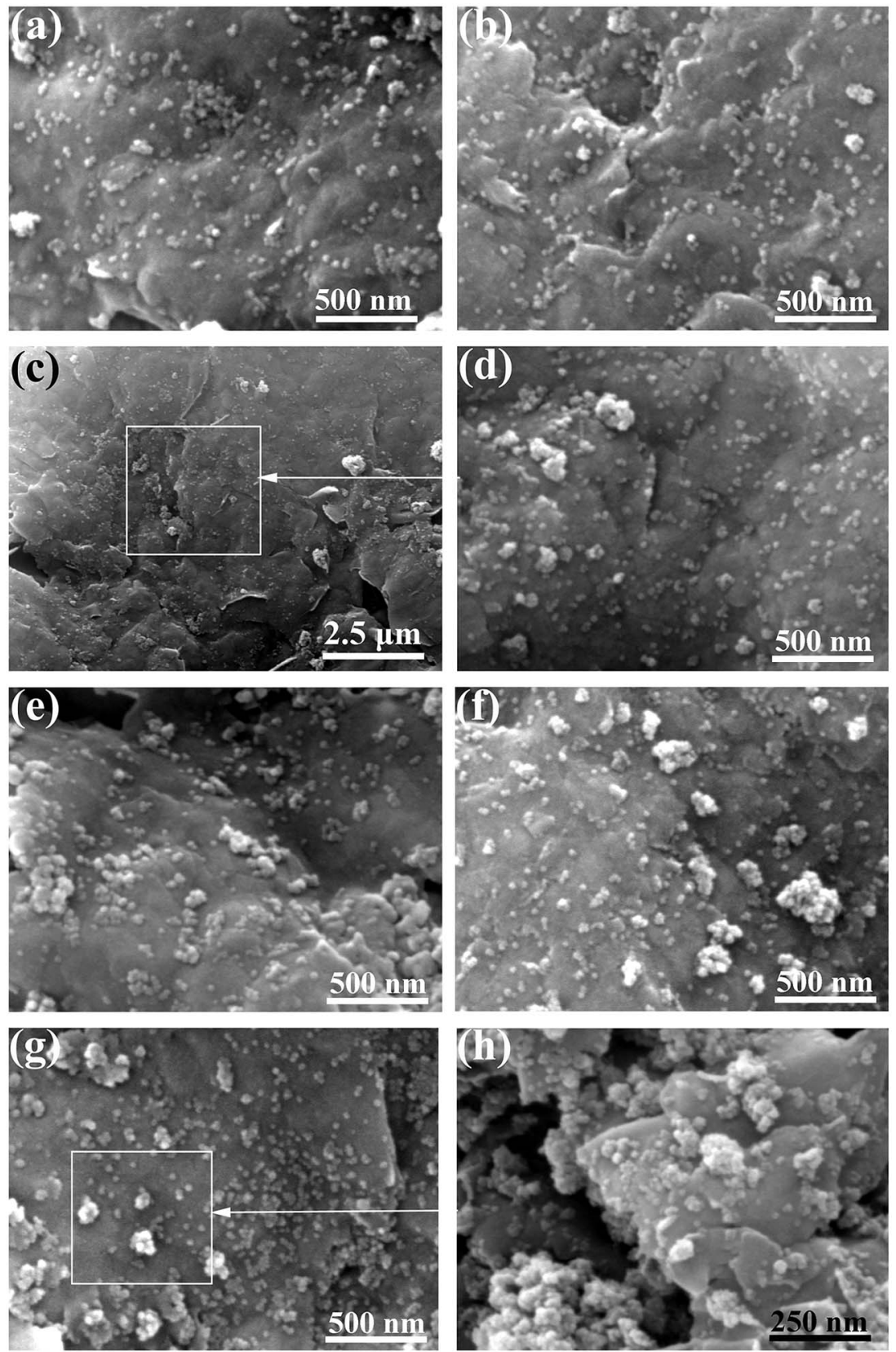

Fig. 7 SEM micrographs of MMT/PQAC $-\mathrm{SiO}_{2}$ nanocomposites with MMT/PQAC $-\mathrm{SiO}_{2}$ weight ratios of (a) 4/1, (b) 3/1, (c, d) 1/1, (e) 1/1.25 (f) 1/2 and $(g, h) 1 / 3$. 

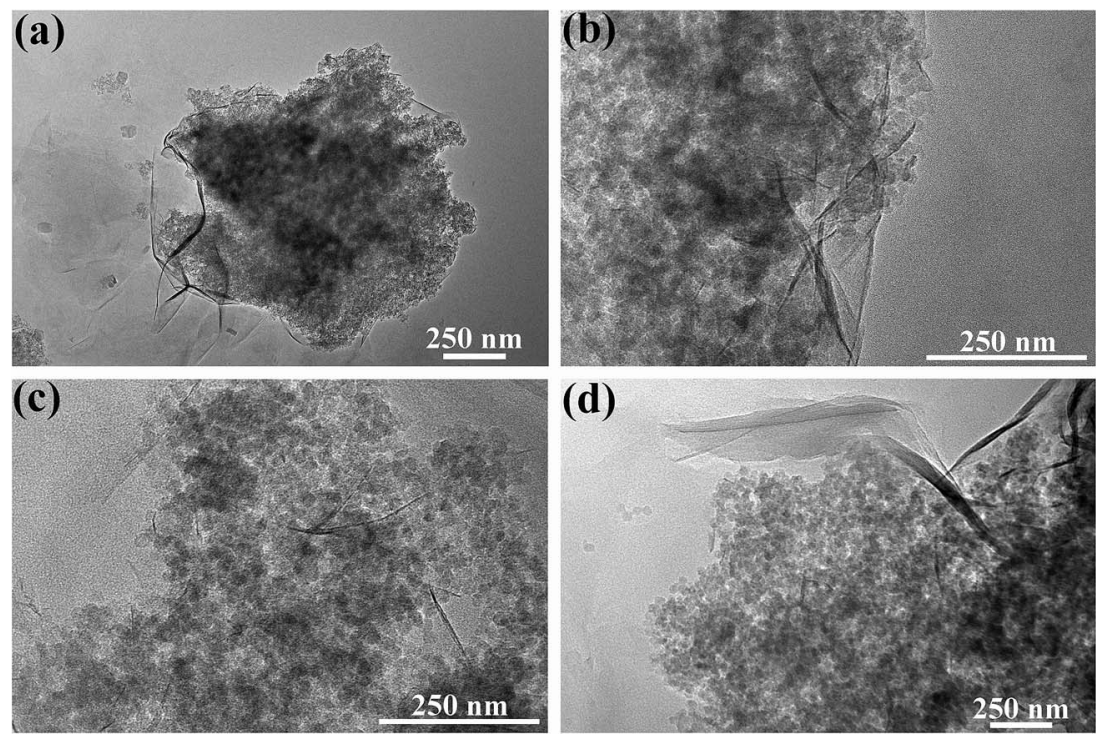

Fig. 8 TEM micrographs of MMT/PQAC $-\mathrm{SiO}_{2}$ nanocomposites with MMT/PQAC- $\mathrm{SiO}_{2}$ weight ratios of (a) 3/1, (b) 1/1, (c) 1/2, and (d) $1 / 3$.
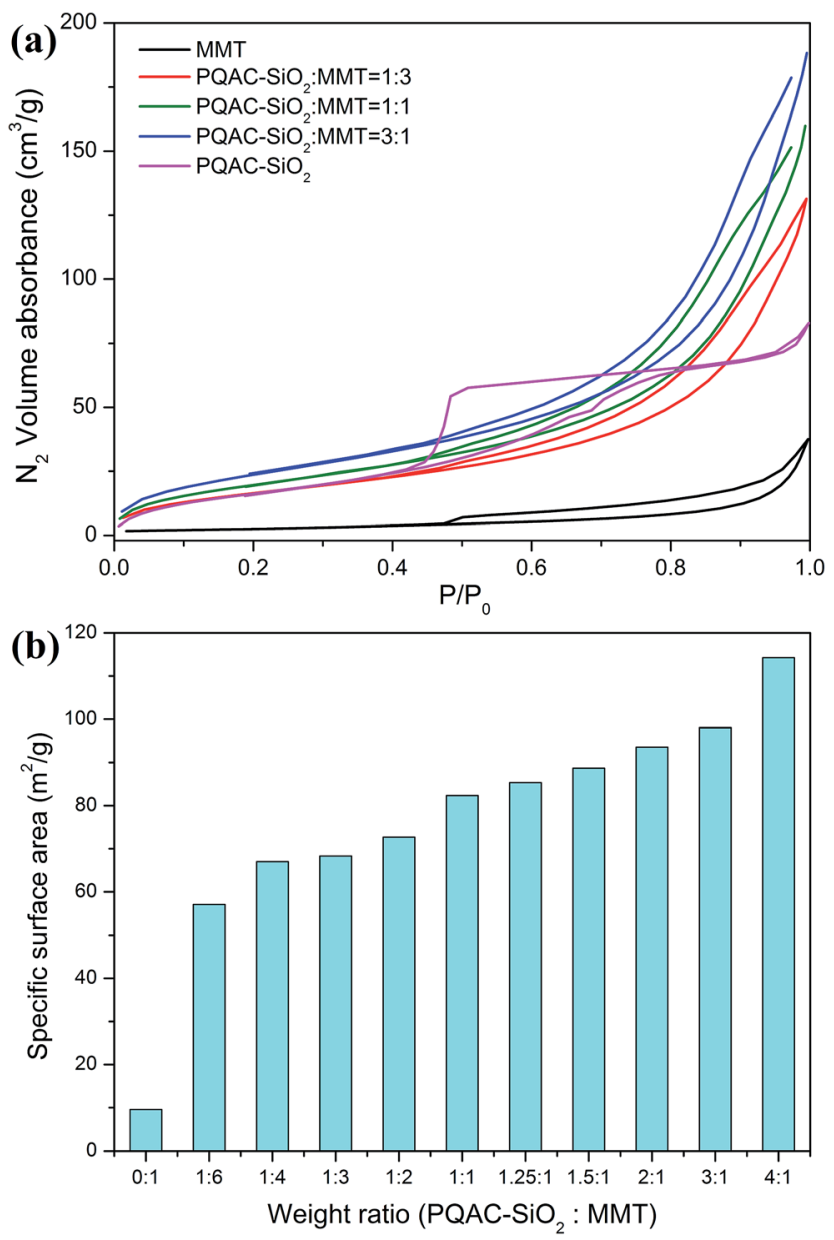

Fig. 9 (a) The $\mathrm{N}_{2}$ adsorption-desorption isotherms and (b) specific surface areas of pure MMT, $\mathrm{PQAC}-\mathrm{SiO}_{2}$ nanoparticles and MMT/ $\mathrm{PQAC}-\mathrm{SiO}_{2}$ nanocomposites.
Deming-Teller (BDDT) classification. Such a characteristic isotherm is ascribed to the existence of microporous structure caused by the aggregation of $\mathrm{PQAC}-\mathrm{SiO}_{2}$ nanoparticles. ${ }^{43}$ On the other hand, both pristine MMT and MMT/PQAC-SiO 2 nanocomposites exhibit a gradual $\mathrm{N}_{2}$-absorption increase with pressure in the relative pressure $\left(P / P_{0}\right)$ range of $0-0.48$ and then a desorption delay in the $P / P_{0}$ range of $0.48-0.9$ in their $\mathrm{N}_{2}$ adsorption-desorption isotherms, which can be assigned to a Type IV isotherms with a typical $\mathrm{H} 4$ hysteresis loop. ${ }^{4}$ The MMT/PQAC-SiO ${ }_{2}$ nanocomposites are found to present a monolayer adsorption on the surfaces of MMT in the initial stage of isotherms at low $P / P_{0}$ values and then a multilayer adsorption in the $P / P_{0}$ range of $0.05-0.48$. When the pressure increases to high values $\left(P / P_{0}=0.48-0.9\right)$, a steep increment appears due to capillary condensation within the interlayers of MMT/PQAC-SiO ${ }_{2}$ nanocomposites followed by a saturation of $\mathrm{N}_{2}$ gas as the interlayers were filled with liquid nitrogen. These results confirm the existence of a layered structure within the $\mathrm{MMT} / \mathrm{PQAC}-\mathrm{SiO}_{2}$ nanoparticles. Furthermore, it is noticeable in Fig. 9b that the specific surface areas of $\mathrm{MMT} / \mathrm{PQAC}-\mathrm{SiO}_{2}$ nanocomposites exhibit a significant increase compared to that of pristine MMT, and the higher the weigh fraction of PQAC$\mathrm{SiO}_{2}$ nanoparticles., the larger the specific surface areas. This phenomenon is attributed to the introduction of $\mathrm{PQAC}-\mathrm{SiO}_{2}$ nanoparticles with a larger surface area as well as the enlargement of interlayer space of MMT.

\subsection{Thermal stability}

The thermal stabilities of $\mathrm{MMT} / \mathrm{PQAC}-\mathrm{SiO}_{2}$ nanocomposites were investigated by TGA, and the resulting TGA and derivate TG (DTG) thermograms are depicted in Fig. 10. It is observed that pristine MMT exhibits two distinct weight losses at 25$100{ }^{\circ} \mathrm{C}$ and $600-650{ }^{\circ} \mathrm{C}$ due to the desorption of surface free water and interlayer water, respectively. ${ }^{\mathbf{4 4}}$ On the other hand, MMT/PQAC- $\mathrm{SiO}_{2}$ nanocomposites are found to exhibit a two- 

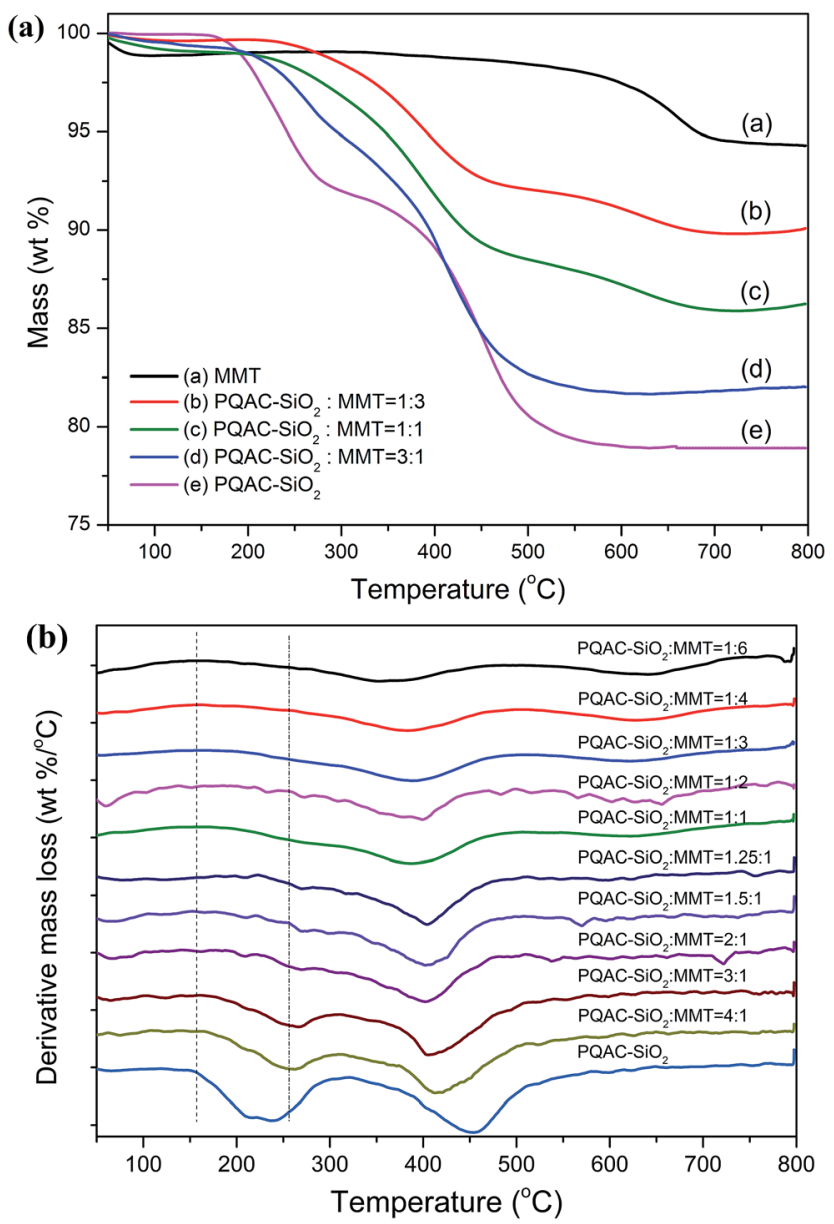

Fig. 10 (a) TGA and (b) DTG thermograms of MMT, PQAC-SiO 2 nanoparticles and MMT/PQAC $-\mathrm{SiO}_{2}$ nanocomposites.

step decomposition behavior in the presence of $\mathrm{PQAC}-\mathrm{SiO}_{2}$ nanoparticles. The first degradation is attributed to the decomposition of quaternary ammonium ions on the PQAC$\mathrm{SiO}_{2}$ nanoparticles and the second one is ascribed to the pyrolysis of alkane chains of PQAC as mentioned previously. However, the initial weight loss at $25-100{ }^{\circ} \mathrm{C}$ is not observed from the MMT/PQAC-SiO ${ }_{2}$ nanocomposites, indicating that these nanocomposites have been dried completely. It is noteworthy in Fig. 10a that the initial decomposition temperature of $\mathrm{MMT} / \mathrm{PQAC}-\mathrm{SiO}_{2}$ nanocomposites is improved significantly to approximately $200{ }^{\circ} \mathrm{C}$ compared to that of pristine PQAC-SiO ${ }_{2}$ nanoparticles. Such an improvement in initial decomposition temperature is ascribed to the fact that the $\mathrm{PQAC}-\mathrm{SiO}_{2}$ nanoparticles are well protected through an interlayer effect when intercalated into the interlayers of MMT. ${ }^{37,41}$ Moreover, as depicted in Fig. 10b, both the initial decomposition temperature and the maximum rapid degradation temperature tend to increase with increasing the weight fraction of $\mathrm{PQAC}-\mathrm{SiO}_{2}$ nanoparticles. Meanwhile, it is interesting to note that the weight loss of interlayer water gradually disappears with increasing the weight fraction of $\mathrm{PQAC}-\mathrm{SiO}_{2}$ nanoparticles. This may be due to the fact that the formation of pillaring structure by $\mathrm{PQAC}-\mathrm{SiO}_{2}$ nanoparticles enlarges the interlayer spacing and thus makes the interlayer water easily evaporate during the drying process. These results suggest that the intercalation of PQAC-SiO ${ }_{2}$ nanocomposites into MMT can effectively improve the thermal stability of $\mathrm{MMT} / \mathrm{PQAC}-\mathrm{SiO}_{2}$ nanocomposites through the interlayer effect and therefore enhances the durability and reliability of PQAC-SiO ${ }_{2}$ nanoparticles when used as an antibacterial agent.

\subsection{Antibacterial performance}

A time-dependent antibacterial study was conducted to evaluate the antibacterial activities of $\mathrm{MMT} / \mathrm{PQAC}-\mathrm{SiO}_{2}$ nanocomposites against $E$. coli and $S$. aureus as the model microorganisms of Gram-negative and Gram-positive bacteria, respectively. A colony counting method was adopted to determine the inhibition of bacteria growth at different incubation time under the antibacterial action of $\mathrm{MMT} / \mathrm{PQAC}-\mathrm{SiO}_{2}$ nanocomposites. Fig. 11 shows the survival rates of two bacteria as a function of contact time upon exposure to $\mathrm{MMT} / \mathrm{PQAC}-\mathrm{SiO}_{2}$ nanocomposites. It seems that the $\mathrm{MMT} / \mathrm{PQAC}-\mathrm{SiO}_{2}$ nanocomposites exhibit the almost same antibacterial effect on $E$. coli and S. aureus. As shown in Fig. 11, E. coli and S. aureus show a drastic decrease in survival rate during the initial four hours when contacting the nanocomposites, and then, their decrements tend to decline. After contacting with the nanocomposites for $7 \mathrm{~h}$, both $E$. coli and $S$. aureus were exterminated completely. This sterilization process can also be observed visibly from the digital photographs of the colonies of bacteria incubated on agar plates in Fig. 12. These photographs clearly demonstrate a significant reduction in the number of bacterial colonies with an increase of contact time. This result confirms an excellent antibacterial effectiveness of $\mathrm{MMT} / \mathrm{PQAC}-\mathrm{SiO}_{2}$ nanocomposites against Gram-positive and Gram-negative bacteria.

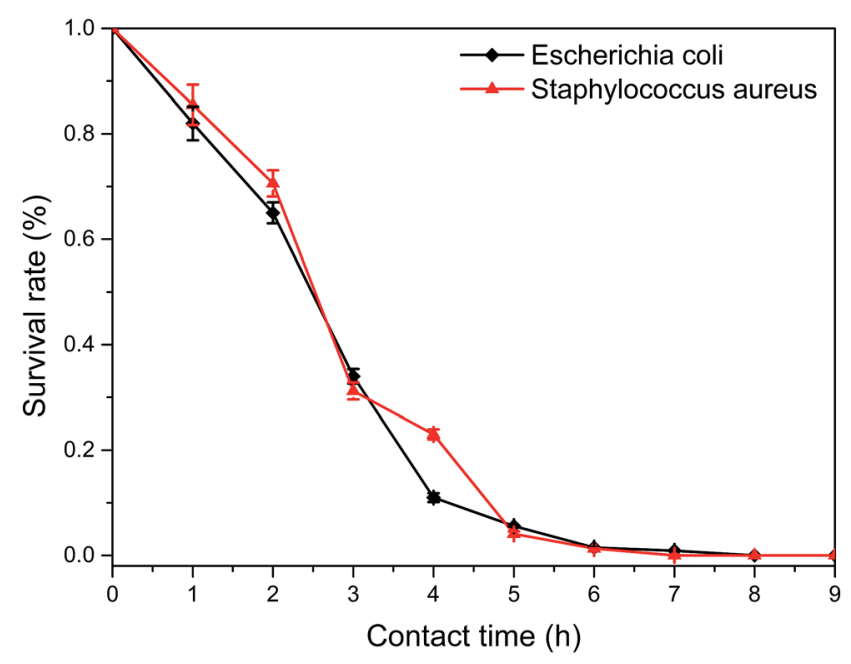

Fig. 11 Plots of the survival rates of model microorganisms as a function of contact time with the MMT/PQAC $-\mathrm{SiO}_{2}$ nanocomposites as an antibacterial agent. 


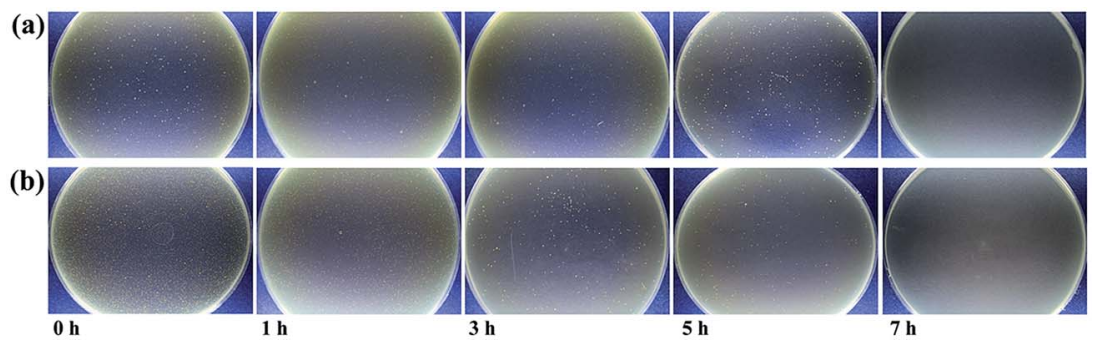

Fig. 12 Optical photographs of the Petri dishes loading (a) E. coli and (b) S. aureus at different contact time.

\section{Conclusions}

The positively charged PQAC- $\mathrm{SiO}_{2}$ nanoparticles were synthesized by grafting PQAC onto the surface of silica nanoparticles and their chemical composition and structure were confirmed by FTIR spectroscopy, XPS and TGA. The layer-structured MMT/ $\mathrm{PQAC}_{-} \mathrm{SiO}_{2}$ nanocomposites were successfully prepared by intercalating $\mathrm{PQAC}-\mathrm{SiO}_{2}$ nanoparticles into the interlayers of MMT through an electrostatic self-assembly method. The layered structure of $\mathrm{MMT} / \mathrm{PQAC}-\mathrm{SiO}_{2}$ nanocomposites was confirmed by XRD patterns, $\mathrm{N}_{2}$ adsorption-desorption analysis, SEM and TEM, and the optimum weight ratio of $2 / 1$ between PQAC-SiO ${ }_{2}$ nanoparticles and MMT was obtained on the basis of these characterization results. Further studies by $\mathrm{N}_{2}$ adsorption-desorption experiments of nanocomposites confirmed the existence of a layered structure within the MMT/ PQAC-SiO ${ }_{2}$ nanocomposites. The intercalation of $\mathrm{PQAC}-\mathrm{SiO}_{2}$ nanoparticles into MMT also improved their thermal stability, resulting in a remarkable increment in onset thermal decomposition from $160{ }^{\circ} \mathrm{C}$ to $200{ }^{\circ} \mathrm{C}$ in comparison with $\mathrm{PQAC}-\mathrm{SiO}_{2}$ nanoparticles. The $\mathrm{MMT} / \mathrm{PQAC}-\mathrm{SiO}_{2}$ nanocomposites also exhibited an excellent antibacterial capability against $E$. coli and $S$. aureus as the model microorganisms of Gram-negative and Gram-positive, respectively.

\section{Acknowledgements}

The financial support from the National Key R\&D Program of China (2014BAE12B03) is gratefully acknowledged.

\section{References}

1 H. H. Murray, Appl. Clay Sci., 2000, 17, 207-221.

2 M. Kotal and A. K. Bhowmick, Prog. Polym. Sci., 2015, 51, 127-187.

3 A. C. Lopes, P. Martins and S. Lanceros-Mendez, Prog. Surf. Sci., 2014, 89, 239-277.

4 B. Li, H. Mao, X. Li, W. Ma and Z. Liu, J. Colloid Interface Sci., 2009, 336, 244-249.

5 M. Polverejan, Y. Liu and T. J. Pinnavaia, Chem. Mater., 2002, 14, 2283-2288.

6 J. Ahenach, P. Cool and E. F. Vansant, Phys. Chem. Chem. Phys., 2000, 2, 5750-5755.

7 A. De Stefanis and A. A. G. Tomlinson, Catal. Today, 2006, 114, 126-141.
8 N. Bitinis, M. Hernandez, R. Verdejo, J. M. Kenny and M. A. Lopez-Manchado, Adv. Mater., 2011, 23, 5229-5236.

9 Y. S. Han and S. Yamanaka, J. Solid State Chem., 2006, 179, 1146-1153.

10 Y. Kameshima, Y. Tamura, A. Nakajima and K. Okada, Appl. Clay Sci., 2009, 45, 20-23.

11 N. Khalfaoui-Boutoumi, H. Boutoumi, H. Khalaf and B. David, Appl. Clay Sci., 2013, 80, 56-62.

12 H. Mao, B. Li, L. Yue, L. Wang, J. Yang and X. Gao, Appl. Clay Sci., 2011, 53, 676-683.

13 M. C. Margarita Darder and E. Ruiz-Hitzky, Chem. Mater., 2003, 2003, 3774-3780.

14 J. J. Lin, W. C. Lin, S. D. Li, C. Y. Lin and S. H. Hsu, ACS Appl. Mater. Interfaces, 2013, 5, 433-443.

15 M. Lavorgna, I. Attianese, G. G. Buonocore, A. Conte, M. A. Del Nobile, F. Tescione and E. Amendola, Carbohydr. Polym., 2014, 102, 385-392.

16 G. A. Sotiriou, A. Meyer, J. T. Knijnenburg, S. Panke and S. E. Pratsinis, Langmuir, 2012, 28, 15929-15936.

17 L. Zhou, H. Chen, X. Jiang, F. Lu, Y. Zhou, W. Yin and X. Ji, J. Colloid Interface Sci., 2009, 332, 16-21.

18 R. K. Khalil, World J. Microbiol. Biotechnol., 2013, 29, 18391850.

19 E. Obłąk, A. Piecuch, A. Krasowska and J. Łuczyński, Microbiol. Res., 2013, 168, 630-638.

20 M. Tischer, G. Pradel, K. Ohlsen and U. Holzgrabe, ChemMedChem, 2012, 7, 22-31.

21 K. Hegstad, S. Langsrud, B. T. Lunestad, A. A. Scheie, M. Sunde and S. P. Yazdankhah, Microb. Drug Resist., 2010, 16, 91-104.

22 B. Dizman, M. O. Elasri and L. J. Mathias, J. Polym. Sci., Part A: Polym. Chem., 2006, 44, 5965-5973.

23 A. W. Smith, Adv. Drug Delivery Rev., 2005, 57, 1539-1550.

24 X. Ye, J. Feng, J. Zhang, X. Yang, X. Liao, Q. Shi and S. Tan, Colloids Surf., B, 2017, 149, 322-329.

25 A. Dong, Q. Zhang, T. Wang, W. Wang, F. Liu and G. Gao, J. Phys. Chem. C, 2010, 114, 17298-17303.

26 B. Song, C. Wu and J. Chang, Acta Biomater., 2012, 8, 19011907.

27 G. S. Alvarez, C. Helary, A. M. Mebert, X. Wang, T. Coradin and M. F. Desimone, J. Mater. Chem. B, 2014, 2, 4660-4670.

28 N. Y. Hebalkar, S. Acharya and T. N. Rao, J. Colloid Interface Sci., 2011, 364, 24-30.

29 N. Hao, X. Chen, K. W. Jayawardana, B. Wu, M. Sundhoro and M. Yan, Biomater. Sci., 2016, 4, 87-91. 
30 Q. Qiu, T. Liu, Z. Li and X. Ding, J. Mater. Chem. B, 2015, 3, 7203-7212.

31 X. S. Zhou, Y. X. Yin, L. J. Wan and Y. G. Guo, Adv. Energy Mater., 2012, 2, 1086-1090.

32 F. Z. Tu, X. Xu, P. Z. Wang, L. Si, X. S. Zhou and J. C. Bao, J. Phys. Chem. C, 2017, 121, 3261-3269.

33 A. B. Bourlinos, R. Herrera, N. Chalkias, D. D. Jiang, Q. Zhang, L. A. Archer and E. P. Giannelis, Adv. Mater., 2005, 17, 234-237.

34 X. Zhang, X. Wang and D. Wu, Energy, 2016, 111, 498-512.

35 D. Q. Wu, H. C. Cui, J. Zhu, X. H. Qin and T. Xie, J. Mater. Chem. B, 2016, 4, 2606-2613.

36 F. Li, X. Wang and D. Wu, Energy Convers. Manage., 2015, 106, 873-885.

37 T. F. Tseng and J. Y. Wu, J. Mater. Chem. C, 2009, 113, 1303613044.
38 N. Andreu, D. Flahaut, R. Dedryvere, M. Minvielle, H. Martinez and D. Gonbeau, ACS Appl. Mater. Interfaces, 2015, 7, 6629-6636.

39 K. Fujii, S. Hayashi and H. Kodama, Chem. Mater., 2003, 15, 1189-1197.

40 Y. Zheng and A. Zaoui, Solid State Ionics, 2011, 203, 80-85.

41 A. Hilonga, J. K. Kim, P. B. Sarawade, D. V. Quang, G. N. Shao, G. Elineema and H. T. Kim, Mater. Lett., 2012, 80, 168-170.

42 X. Liu, Y. C. Du, L. Y. Hu, X. S. Zhou, Y. F. Li, Z. H. Dai and J. C. Bao, J. Phys. Chem. C, 2015, 119, 5848-5854.

43 N. Ahmad, S. T. Hussain, B. Muhammad, N. Ali, S. M. Abbas and Z. Ali, Prog. Nat. Sci.: Mater. Int., 2013, 23, 374-381.

44 B. Wu, S. Qi and X. Wang, Polym. Test., 2010, 29, 717-722. 